\title{
Interactive comment on "Prediction of source contributions to urban background PM10 concentrations in European cities: a case study for an episode in December 2016 - Part.1 The country contributions” by Matthieu Pommier et al.
}

Matthieu Pommier et al.

matthieup@met.no

Received and published: 22 September 2019

The comment was uploaded in the form of a supplement:

https://www.geosci-model-dev-discuss.net/gmd-2019-87/gmd-2019-87-AC4-

supplement.pdf 